\title{
Glucocorticoid receptor DNA methylation and childhood trauma in chronic fatigue syndrome patients
}

\author{
Running title: GR DNA methylation and childhood trauma in CFS
}

Elise Beau Vangeel ${ }^{1,2}\left({ }^{*}\right), \mathrm{PhD}$, Stefan Kempke ${ }^{1}$, PhD, Jelena Bakusic ${ }^{3}, \mathrm{MSc}$, Lode Godderis ${ }^{3,4}, \mathrm{MD}$, PhD, Patrick Luyten ${ }^{5,6}, \mathrm{PhD}$, Leen Van Heddegem ${ }^{7}, \mathrm{MSc}$, Veerle Compernolle ${ }^{7}, \mathrm{MD}$, PhD, Philippe Persoons ${ }^{8}, \mathrm{MD}, \mathrm{PhD}$, Diether Lambrechts ${ }^{9,10}, \mathrm{PhD}$, Benedetta Izzi ${ }^{11}$, PhD, Kathleen Freson ${ }^{2}, \mathrm{PhD}$, Stephan Claes ${ }^{1,8}, \mathrm{MD}, \mathrm{PhD}$

Affiliations:

1: Genetic Research About Stress and Psychiatry (GRASP), Department of Neurosciences, KU Leuven, Leuven, Belgium

2: Center for Molecular and Vascular Biology (CMVB), Department of Cardiovascular Sciences, KU Leuven, Leuven, Belgium

3: Environment and Health, Department of Public Health and Primary Care, KU Leuven, Leuven, Belgium

4: IDEWE, External Service for Prevention and Protection at Work, Heverlee, Belgium

5: Faculty of Psychology and Educational Sciences, KU Leuven, Leuven, Belgium

6: Research Department of Clinical, Educational and Health Psychology, University College London, London, UK

7: Blood Service, Belgian Red Cross-Flanders, Mechelen, Belgium

8: University Psychiatric Center, Leuven, Belgium

9: Laboratory of Translational Genetics, Department of Human Genetics, KU Leuven, Leuven, Belgium

10: VIB Center for Cancer Biology, VIB, Leuven, Belgium

11: Department of Epidemiology and Prevention, IRCCS Neurologico Mediterraneo Neuromed, Pozzilli, Italy

* Corresponding author:

Elise Beau Vangeel

Elisebeauvangeel@gmail.com

Research Group Psychiatry

Herestraat 49, 3000 Leuven, Belgium

Tel: +3216340548

Manuscript word count Introduction - Discussion: 3637

Tables: 3 


\section{ABSTRACT}

Objective: Although the precise mechanisms are not yet understood, previous studies have suggested that chronic fatigue syndrome (CFS) is associated with hypothalamic-pituitary-adrenal (HPA) axis dysregulation and trauma in early childhood. Consistent with findings suggesting that early life stressinduced DNA methylation changes may underlie dysregulation of the HPA axis, we previously found evidence for the involvement of glucocorticoid receptor (GR) gene (NR3C1) methylation in whole blood of CFS patients.

Methods: In the current study, we assessed NR3C1-1F region DNA methylation status in peripheral blood from a new and independent sample of 80 female CFS patients and 91 female controls. In CFS patients, history of childhood trauma subtypes was evaluated using the Childhood Trauma Questionnaire short form (CTQ-SF).

Results: Although absolute methylation differences were small, the present study confirms our previous findings of NR3C1-1F DNA hypomethylation at several CpG sites in CFS patients as compared to controls. Following multiple testing correction, only $\mathrm{CpG}_{-} 8$ remained significant (DNA methylation difference: $1.3 \%$ versus $1.5 \%, \mathrm{p}<0.001)$. In addition, we found associations between DNA methylation and severity of fatigue as well as with childhood emotional abuse in CFS patients, although these findings were not significant after correction for multiple testing.

Conclusions: In conclusion, we replicated findings of NR3C1-1F DNA hypomethylation in CFS patients versus controls. Our results support the hypothesis of HPA axis dysregulation and enhanced GR sensitivity in CFS.

Key words: childhood trauma; chronic fatigue syndrome; DNA methylation; glucocorticoid receptor; HPA axis; NR3C1 
List of abbreviations

$\mathrm{BPD}=$ borderline personality disorder

$C D C=$ US Centers for Disease Control and Prevention

CFS = chronic fatigue syndrome

$\mathrm{CTQ}-\mathrm{SF}=$ childhood trauma questionnaire short form

FKBP5 $=$ FK506 binding protein 5

$\mathrm{GR}=$ glucocorticoid receptor

HADS = Hospital Anxiety and Depression Scale

HPA = hypothalamic-pituitary-adrenal

NGFI-A = nerve growth factor-inducible protein A

$\mathrm{NR} 3 \mathrm{C} 1$ = nuclear receptor subfamily 3 group $\mathrm{C}$ member 1

PTSD = post-traumatic stress syndrome

SCID = Structured Clinical Interview for Diagnostic and Statistical Manual for Mental Disorders 


\section{INTRODUCTION}

Chronic fatigue syndrome (CFS) is a highly debilitating syndrome involving severe and long-lasting fatigue not due to known medical causes, accompanied by four or more of the following symptoms, i.e. impaired memory or concentration, headaches, unrefreshing sleep, post-exertional malaise, sore throat, joint and muscle pain [1]. Diagnosis of CFS is disproportionally higher in women than in men [2]. Reported CFS prevalence worldwide ranges from $0.2 \%$ up to $6.41 \%[3,4]$, while a recent metaanalysis reports a prevalence of $0.76 \%$ for clinically assessed CFS [5]. The disorder is associated with extremely high health-care costs, for instance the annual productivity costs of CFS in the UK is estimated at approximately $f 100$ million [6].

Stress, and in particular chronic stress, may play a role in the etiology and maintenance of CFS [7-10]. Indeed, studies have suggested that adverse events may cause dysregulation of the stress system, and the hypothalamic-pituitary-adrenal (HPA) axis, ultimately resulting in an altered stress system reponse $[8,11,12]$. This is in agreement with the concept of "allostatic load", as first formulated by McEwen and Stellar in 1993 [13]. Allostatic load refers to the effects of the "wear-and-tear" of cumulative life stress on the stress system, which can ultimately accelerate disease development [14]. It is suggested that over time, chronic stress may lead to a blunted HPA axis response [15]. There is indeed evidence for a lower cortisol response and hypocortisolism in at least a subsample of CFS patients, reflecting a loss of adaptability of the main neurobiological stress response system after a prolonged period of chronic stress [16-18].

The glucocorticoid receptor (GR) may be involved in the etiology of CFS, since it plays a key role in the HPA axis response by exerting a negative feedback mechanism. More specifically, when cortisol is secreted by the HPA axis, its binding to the GR in the hypothalamus and pituitary gland will subsequently attenuate the stress response, preventing the cascade from continuing disproportionately [19]. Changes in GR sensitivity could therefore alter the stress response and potentially influence the susceptibility for stress-related disorders. Enhanced GR sensitivity has been 
described in CFS patients during dexamethasone suppression tests [20-22]. DNA methylation changes in the GR gene (NR3C1) exon 1F promoter region may be a potential mechanism. Indeed, NR3C1-1F hypomethylation was found in peripheral blood mononuclear cells of combat veterans with posttraumatic stress disorder (PTSD), which was additionally associated with cortisol response following dexamethasone administration [23]. Moreover, in a previously published study we showed that HPA axis response was significantly associated with DNA methylation levels at the NR3C1-1F region in a study sample of 76 female CFS patients [24]. Furthermore, this patient population presented with significant NR3C1-1F hypomethylation in whole blood compared to healthy female controls. An association with childhood trauma within the CFS patient group was not found, although DNA methylation in the examined region has previously been linked to early adversity (for Review: [25]). For instance, McGowan et al. showed NR3C1-1F DNA methylation differences in hippocampal brain tissue of suicide completers who were exposed to childhood abuse, versus suicide completers without childhood abuse [26]. An association of childhood trauma history with NR3C1-1F methylation was also shown in peripheral tissues, for instance in blood samples from bipolar and depressed patients [27] and saliva from depressed patients [28].

There is increasing evidence from both cross-sectional $[10,17,29-33]$ and prospective studies [34-40] that childhood trauma is an important risk factor for CFS. Early life stress exposure may influence DNA methylation of HPA axis related genes, subsequently altering HPA reactivity, which in turn may represent a risk factor for stress-related disorders, including CFS [41].

In the current study, we intended to replicate and extend our previous finding of NR3C1-1F hypomethylation in CFS patients by examining the $1 \mathrm{~F}$ promoter region in more detail, and in a larger study sample. Specifically, we investigated the association between NR3C1-1F methylation in whole blood and CFS symptom severity in carefully screened CFS patients as compared to a control group, in addition to using a well-validated tool to retrospectively assess childhood trauma. 


\section{MATERIALS AND METHODS}

\subsection{Study participants}

The study was conducted between December 2014 and February 2016. Whole blood samples from female patients diagnosed with CFS ( $n=80)$ according to the US Centers for Disease Control and Prevention (CDC) guidelines [1] were obtained at the CFS reference center of the University Hospitals Leuven, Belgium. Alternative medical conditions that might explain fatigue were excluded following a full medical screening by an internist including clinical examinations and laboratory tests. For a detailed overview, see Kempke et al. [17]. Subsequently, patients received a psychiatric evaluation to exclude psychiatric conditions associated with fatigue complaints. The Structured Clinical Interview for Diagnostic and Statistical Manual for Mental Disorders, Fourth Edition (DSM-IV), Axis I Disorders (SCIDI) depression module was administered to exclude all patients with a diagnosis of current depression [42]. Patients did not use corticosteroids and did not have thyroid dysfunction.

Whole blood samples for the female control group $(n=91)$ were derived from voluntary nonremunerated plasma donors fulfilling Belgian eligibility criteria at the Red Cross donor center in Leuven. A blood sample was only taken if the donor did not meet any of the exclusion criteria. The following exclusion criteria were examined for the control participants by self-report questionnaire: having (1) a non-European parent (given the distribution of genetic variants and possible ethnicity-dependent changes in DNA methylation), (2) unremitting fatigue symptoms, (3) unremitting pain symptoms, (4) history of CFS or fibromyalgia, (5) current depressive episode, and (6) lifetime psychiatric hospitalization.

All participants, from the patient as well as the control group, were more than 18 years old and were not pregnant or breastfeeding. Blood samples were collected from all participants in $10 \mathrm{~mL}$ EDTA tubes. Informed consent was obtained from all participants and the study was approved by the University Hospitals Leuven Medical Ethics Committee (B322201421832).

\subsection{Questionnaires}


CFS symptom severity was examined in the patient group using the Checklist Individual Strength (CIS) which consists of four subscales: fatigue, concentration, motivation and physical activity [43]. To assess a history of childhood trauma, CFS patients completed the short version of the Childhood Trauma Questionnaire (CTQ-SF), which has shown good validity in assessing childhood trauma as compared to structured interviews [44]. The CTQ-SF consists of 25 items, assessing exposure to sexual, physical or emotional abuse and physical or emotional neglect before the age of 18 . Each subscale of the questionnaire contains five questions to be answered on a five-point Likert scale. Therefore, the range of scores for each trauma domain is situated between 5 and 25 , while the total score ranges from 25 to 125 [44]. For the childhood trauma subtype frequency table, the same cutoffs were used as in Heim et al. [45]: a score of 13 or higher for emotional abuse, 10 or higher for physical abuse, 8 or higher for sexual abuse, 15 or higher for emotional neglect, and 10 or higher for physical neglect. For correlation analyses, CTQ-SF total and subdomain scores were used as continuous variables. Depressive symptoms in the patient group were measured using the Hospital Anxiety and Depression Scale (HADS), which is a 21 points scale specifically developed to assess depression in medical conditions [46]. CFS patients completed questionnaires at home by logging onto a web-based application of the University Hospitals Leuven ('myNEXUZ'). If patients had no access to internet, a paper-and-pencil version of the questionnaires was provided. Since we aimed to examine patients diagnosed with CFS in more detail regarding childhood trauma, these questionnaires were only administered to the patient group.

\subsection{NR3C1-1F DNA methylation analyses using pyrosequencing and EpiTYPER}

DNA was extracted from peripheral whole blood samples from all participants. From all samples, $1 \mu \mathrm{g}$ of DNA was bisulfite treated using the MethylDetector kit (Active Motif, La Hulpe, Belgium) according to the suggested long cycling protocol [47]. In order to investigate the previously reported differentially methylated CpG sites of NR3C1-1F (CpG_1 until CpG_5) in more detail [24], we carried out pyrosequencing for the DNA region spanning CpG_1 until CpG_8. Data for the remaining CpG sites within the NR3C1-1F region (from CpG_9 until CpG_47) were generated using the EpiTYPER 
methodology. More specifically, to investigate DNA methylation at the individual $\mathrm{CpG}$ sites from CpG_1 until CpG_8, we assessed the DNA methylation status of the NR3C1-1F region that was used by Perroud et al. (2011) by pyrosequencing [27]. We amplified a fragment of $403 \mathrm{bp}$ using forward (5'TTTGAAGTTTTTTTAGAGGG-3') and reverse (5'-CCCCCAACTCCCCAAAAA-3', with 5' biotin) primers (IDT, Leuven, Belgium) using a slight adjustment to the conditions of Perroud et al. (2011), i.e. a PCR cycling program of $94^{\circ} \mathrm{C}$ for 15 minutes, followed by 50 cycles of $94^{\circ} \mathrm{C}$ for 1 minute, $58^{\circ} \mathrm{C}$ for 1 minute, $72^{\circ} \mathrm{C}$ for 1 minute and finally an extension step of 10 minutes at $72^{\circ} \mathrm{C}$. Subsequently, pyrosequencing was performed using the sequencing primer (5'-AGAAAAGAAATTGGAGAAATT-3') on the Pyromark Q24 platform (Qiagen, Hilden, Germany) to assess DNA methylation values.

Additionally, the same bisulfite treated DNA samples were used to carry out the Agena Bioscience EpiTYPER methodology for the NR3C1-1F region spanning CpG_9 until CpG_47, following the guidelines (Agena Bioscience, Hamburg, Germany) [24]. The EpiTYPER method is based on basespecific cleavage followed by mass spectrometry for the sensitive detection of fragment size. The EpiTYPER software then converts these measurements to DNA methylation percentages for each analyzed $\mathrm{CpG}$ unit. Subsequently, quality control was carried out as suggested [48]: $\mathrm{CpG}$ units with a success rate of less than $75 \%$ or a standard deviation between triplicates of more than 0.1 were excluded from the analysis. PCR primers and further technical details to analyze the NR3C1-1F region were described previously [24].

\subsection{Statistical analyses}

Average DNA methylation as well as functional DNA methylation was calculated for the analyzed NR3C1-1F region for the CFS patient group and the control group separately. More specifically, the functional methylation value averages DNA methylation levels only from $\mathrm{CpG}$ sites associated with

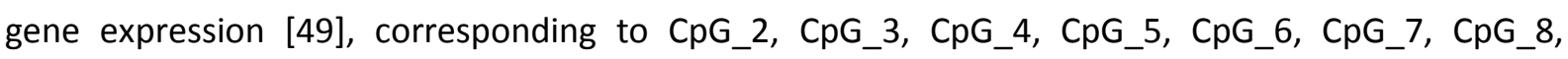
CpG_12.13, CpG_14 and $C p G \_19$ in the current study. The $\mathrm{CpG}$ sites in the NR3C1-1F region consistently show low levels of DNA methylation, causing data to be skewed and therefore not 
normally distributed. For this reason, all statistical analyses were performed using non-parametric tests. Mann-Whitney $U$ tests were used to compare DNA methylation levels between patient and control groups and effect sizes were calculated by dividing the $z$ statistic by the square root of the study sample size (i.e. CFS patients and controls combined) [50]. Spearman correlations were used to examine correlations between DNA methylation and the CIS-20 scale or the CTQ-SF (sub)scales. Tests with a p-value smaller than 0.05 were considered significant. Version 23 of the SPSS statistics software (IBM Corp, Armonk, NY) was used for all analyses. 


\section{RESULTS}

\subsection{Study sample characteristics}

Characteristics of the CFS patient study population can be found in Table 1. The frequency of childhood trauma subdomains in the patient group was summarized in Table 2. Participants in both the CFS patient and the control group were all female and had comparable ages on average (mean +/- SD): $41.36+/-10.74$ and $43.98+/-13.91$ years old, respectively.

\subsection{Comparison of NR3C1-1F DNA methylation between CFS patients and controls}

When comparing DNA methylation levels of CFS patients with controls, we found hypomethylation at CpG_1 $(p=0.010), C p G \_4(p=0.017), C p G \_6(p=0.003), C p G \_7(p=0.006), C p G \_8(p<0.001)$ as well as the functional DNA methylation $(p=0.003$; Table 3$)$. However, when correcting for multiple testing, only CpG_8 remained significantly hypomethylated in CFS patients as compared to control participants. Absolute DNA methylation differences between both groups remained small, while estimated effect sizes were small to moderate and the effect size at CpG_8 was large $(r=0.558)$.

\subsection{NR3C1-1F DNA methylation associations with CFS symptoms and childhood trauma}

Within the CFS patient group, the CIS-20 total scale was correlated with DNA methylation at CpG_17.18 (Spearman's Rho $=-0.241, p=0.034$ ) and CpG_36 (Spearman's Rho $=-0.232, p=0.041)$. Examining both sites in more detail revealed that $\mathrm{CpG}_{3} 36$ was significantly associated with the subscale 'fatigue severity' (Spearman's Rho $=-0.324, p=0.004$; Supplementary Figure S1). None of the NR3C1-1F CpG sites were significantly associated with duration of illness in the CFS patients.

Regarding childhood trauma by CTQ-SF subscales in the CFS patient group, we found that emotional abuse was correlated with DNA methylation at CpG_3 (Spearman's Rho $=0.260, p=0.032$ ) and CpG_47 (Spearman's Rho $=-0.239, p=0.039$; Supplementary Figure S2). These associations were no longer significant after Bonferroni correction for multiple testing. The other CTQ subscales did not appear to be correlated with NR3C1-1F DNA methylation (Supplementary table S1). 


\section{DISCUSSION}

In the current study, we examined the role of NR3C1-1F DNA methylation in CFS and its relation to childhood trauma. DNA methylation analyses were performed on DNA extracted from whole blood samples in a population of female CFS patients and control participants. Two candidate gene focused methods were used to measure DNA methylation in the NR3C1-1F region. As in our previous publication [24], we used EpiTYPER since it is able to measure DNA methylation levels at several CpG units across a relatively large region, e.g. the NR3C1-1F amplicon is 402 base pairs long. However, a disadvantage is that the EpiTYPER technique sometimes cannot discriminate between individual, nearby $\mathrm{CpG}$ sites, grouping them together into units. In our previous publication studying NR3C1-1F methylation in CFS patients, we identified a significant effect especially at the CpG_1.2.3.4.5 unit. In addition to mapping DNA methylation in the NR3C1-1F EpiTYPER amplicon, we were determined to additionally measure this particular region in more detail using pyrosequencing. Although the latter technique is limited in the size of the region it can sequence, it was able to separately measure DNA methylation levels at the $\mathrm{CpG}$ sites in the region. Furthermore, pyrosequencing could additionally detect CpG_6, CpG_7 and CpG_8, which could not be measured by EpiTYPER due to their fragment masses not being in range of the mass spectrometer's detection capacity. In sum, using these complementary techniques provides a broad view on DNA methylation in the NR3C1-1F region (EpiTYPER) in addition to separately measuring $\mathrm{CpG}$ sites 1 until 8 (pyrosequencing).

Consistent with our previously published findings [24], we found NR3C1-1F hypomethylation in blood samples of CFS patients versus controls at the $3^{\prime}$ end of the analyzed NR3C1-1F region (CpG_1 to CpG_8). The previous study identified the CpG unit spanning CpG_1 until CpG_5 as significantly different between CFS patients and healthy control subjects. Correspondingly, the current study finds lower DNA methylation levels in the CFS patient group at CpG_1, CpG_4, CpG_6, CpG_7 and CpG_8. Also the functional DNA methylation, which averages methylation values of $\mathrm{CpG}$ sites associated with NR3C1 exon 1F gene expression [49], was lower in CFS patients, suggesting the involvement of 
functionally relevant $\mathrm{CpG}$ sites. However, after correction for multiple testing, only CpG_8 remained significant. Furthermore, estimated effect sizes were small to moderate for all CpG sites, while CpG_8 showed a large effect size. CpG_8 is located within the $1 \mathrm{~F}$ exon of the NR3C1 gene. There is evidence of an association between DNA methylation at this CpG site and further upstream CpG sites (CpG_1 until CpG_8) and borderline personality disorder (BPD) clinical severity [51]. Previously this region was also suggested to be involved in childhood trauma as shown in a population of BPD patients and major depressive disorder patients [27]. In addition, two CpG sites were significantly correlated with the CFS symptom severity measured by the CIS-20 scale (CpG_17.18 and CpG_36) in the current study. Both CpG sites were negatively correlated, consistent with the hypothesis that more severe CFS symptoms would correspond to a more pronounced NR3C1-1F hypomethylation. The CpG_17.18 site associated with the total CIS-20 score in the current study is located within a putative nerve growth factorinducible protein A (NGFI-A) binding site previously associated with childhood abuse [25]. Finally, CpG_36, which was additionally associated with the CIS-20 'fatigue severity' subscale, lies in a noncanonical NGFI-A binding site. In other studies, DNA methylation at this site was associated with posttraumatic stress syndrome (PTSD) [23] and was predictive of cortisol levels in response to a standardized stress task in females [52], indicating the relevance of this particular site in the HPA axis response to stress.

Furthermore, none of the examined NR3C1-1F CpG sites were associated with duration of CFS symptoms, i.e. whether a patient has suffered from CFS for a longer or shorter period of time. This finding implies that DNA methylation state of this region may not be a consequence of CFS. Combining these findings with our previous work, these results would suggest that CFS pathophysiology is mediated by a decreased NR3C1-1F DNA methylation, which may lead to a higher gene expression. Increased GR gene expression is hypothesized to cause an increased HPA negative feedback resulting in hypocortisolism. It has been suggested that CFS patients may have a decreased HPA axis responsiveness [15]. Hypocortisolism and HPA axis hyporesponsiveness have also been implicated in 
PTSD, suggesting a similar underlying pathophysiological mechanism of these stress-related disorders [53].

Studies have indeed shown the involvement of DNA methylation at the NR3C1-1F region in PTSD as well as other candidate genes implicated in the stress response system. One such gene is the gene encoding for the FK506 binding protein 5 (FKBP5), which is an intracellular inhibitor of GR signaling and acts by impeding GR translocation to the nucleus. Interestingly, pre-treatment NR3C1-1F DNA methylation may predict treatment response in PTSD patients, whereas FKBP5 methylation is associated with recovery in PTSD [54]. Hence future research should focus on FKBP5 as potential gene of interest in CFS studies. Indeed, besides NR3C1 there are likely many genes and pathways that interact and play an important role in CFS pathology. Recently, two genome-wide DNA methylation studies in CFS patients found several significant differentially methylated CpG sites in different genes $[55,56]$

In our previously published study, we did not find a difference in NR3C1-1F DNA methylation between CFS patients who had experienced no/mild versus moderate/severe trauma during childhood [24]. Regarding types of childhood trauma in the current study, we showed a correlation of NR3C1-1F DNA methylation at CpG_3 and CpG_47 with emotional abuse within the CFS patient group. Although the opposite signs of the correlation for both $\mathrm{CpG}$ sites may argue a false positive result, they may also indicate that different regions within the $N R 3 C 1$ gene are associated differently with certain functions and characteristics [25]. Although the results do not remain significant following correction for multiple tests, these trends support the assumption that certain types of childhood trauma, such as emotional trauma, may be important in the etiology of CFS. Indeed, studies have found elevated levels of childhood trauma in population-based as well as in clinical samples of CFS patients [31]. The prevalence of emotional trauma has been found to be particularly high in CFS patients [10] and has been associated with blunted HPA axis response in CFS patients [17]. 
Some limitations of our study should be considered when interpreting the results. To assess CFS symptoms and history of childhood trauma, self-report questionnaires were used, which may be subject to reporting bias. Yet, studies have provided validity for the CTQ-SF in assessing childhood trauma and, if anything, underestimates rather than overestimates the prevalence of trauma [57]. Our study design did not allow us to obtain childhood trauma information or depressive symptoms scores from the control group. The aim of our study design was to examine NR3C1 DNA methylation differences and the association with childhood trauma within the CFS patient group. For this reason, we were not able to compare childhood trauma frequencies or depressive symptoms between both groups. Furthermore, due to the cross-sectional nature of the study we cannot make any claims on causality. Disentangling cause and consequence seems to be an important issue for future research. Although the current study lacks biological measurements such as mRNA levels or HPA axis response tests to provide evidence for functional significance of our DNA methylation changes, our previously published study indicates a link between HPA axis response and the DNA hypomethylation of NR3C11F [24]. The small absolute DNA methylation differences identified in the current study correspond to differences found in previous studies examining stress-related disorders [25]. Though small in absolute values, our findings at $\mathrm{CPG}_{-} 8$ showed a large effect size, while small to moderate effect sizes were found at other investigated $\mathrm{CpG}$ sites. Furthermore, DNA methylation differences of small magnitude can be functionally relevant [58]. Future prospective studies are indeed necessary to elucidate the exact biological function of NR3C1-1F DNA methylation in CFS pathophysiology. Finally, it is imperative that the associations between methylation, symptom severity and childhood trauma reported in the current study should be interpreted with caution, since these correlations did not withstand multiple testing correction. The reported NR3C1-1F hypomethylation comprises small changes in absolute DNA methylation levels between patients and controls, which we cannot directly link to functional changes with the current study design. However, it should be noted that similar small changes in NR3C1-1F DNA methylation have previously been reported in several studies [25], while several studies report an association with gene expression or functional HPA axis tests [23, 49, 59-63]. Additionally, our 
measures of DNA methylation showed small standard errors indicating that the NR3C1 promoter region is tightly regulated. It is therefore suggested that gene transcription can be considerably altered by small methylation changes at crucial CpG sites of the NR3C1 promoter region [25].

In summary, we replicated our findings of NR3C1-1F DNA hypomethylation in CFS patients. Furthermore, there was some evidence for an association between DNA methylation at this region and childhood trauma in CFS patients. To our knowledge, only few studies have examined DNA methylation in CFS to this date $[55,56]$. Hence, there is a need for further prospective research on the interactions between exposure to adversity, DNA methylation and CFS pathophysiology.

5. Funding

This project was supported by the Fund for Scientific Research Flanders (FWO; grant number ELGC5778-G.0A69.13). S.C. is a Senior Clinical Researcher supported by the Fund for Scientific Research Flanders (1800411N). B.I. is a Fellow of the Umberto Veronesi Foundation, Milan, Italy. 


\section{REFERENCES}

[1] K. Fukuda, The Chronic Fatigue Syndrome: A Comprehensive Approach to Its Definition and Study, Annals of Internal Medicine 121(12) (1994) 953-953.

[2] L.A. Jason, N. Porter, M. Brown, V. Anderson, A. Brown, J. Hunnell, A. Lerch, CFS: A Review of Epidemiology and Natural History Studies, Bull IACFS ME 17(3) (2009) 88-106.

[3] Y.M. Yiu, M.Y. Qiu, [A preliminary epidemiological study and discussion on traditional Chinese medicine pathogenesis of chronic fatigue syndrome in Hong Kong], J Chin Integr Med / Zhong Xi Yi Jie He Xue Bao 3(5) (2005) 359-62.

[4] L.C. Nacul, E.M. Lacerda, D. Pheby, P. Campion, M. Molokhia, S. Fayyaz, J.C. Leite, F. Poland, A. Howe, M.L. Drachler, Prevalence of myalgic encephalomyelitis/chronic fatigue syndrome (ME/CFS) in three regions of England: a repeated cross-sectional study in primary care, BMC Med 9 (2011) 91.

[5] S. Johnston, E.W. Brenu, D. Staines, S. Marshall-Gradisnik, The prevalence of chronic fatigue syndrome/ myalgic encephalomyelitis: a meta-analysis, Clin Epidemiol 5 (2013) 105-10.

[6] S.M. Collin, E. Crawley, M.T. May, J.A. Sterne, W. Hollingworth, U.C.M.N.O. Database, The impact of CFS/ME on employment and productivity in the UK: a cross-sectional study based on the CFS/ME national outcomes database, BMC Health Serv Res 11 (2011) 217.

[7] U.M. Nater, E. Maloney, C. Heim, W.C. Reeves, Cumulative life stress in chronic fatigue syndrome, Psychiatry Res 189(2) (2011) 318-20.

[8] B. Van Houdenhove, F. Van Den Eede, P. Luyten, Does hypothalamic-pituitary-adrenal axis hypofunction in chronic fatigue syndrome reflect a 'crash' in the stress system?, Med Hypotheses 72(6) (2009) 701-5. 
[9] P. Luyten, S. Kempke, P. Van Wambeke, S. Claes, S.J. Blatt, B. Van Houdenhove, Self-critical perfectionism, stress generation, and stress sensitivity in patients with chronic fatigue syndrome: relationship with severity of depression, Psychiatry 74(1) (2011) 21-30.

[10] S. Kempke, P. Luyten, S. Claes, P. Van Wambeke, P. Bekaert, L. Goossens, B. Van Houdenhove, The prevalence and impact of early childhood trauma in Chronic Fatigue Syndrome, J Psychiatr Res 47(5) (2013) 664-9.

[11] F. Van Den Eede, G. Moorkens, B. Van Houdenhove, P. Cosyns, S.J. Claes, Hypothalamic-pituitaryadrenal axis function in chronic fatigue syndrome, Neuropsychobiology 55(2) (2007) 112-20.

[12] C. Tomas, J. Newton, S. Watson, A review of hypothalamic-pituitary-adrenal axis function in chronic fatigue syndrome, ISRN Neurosci 2013 (2013) 784520.

[13] B.S. McEwen, E. Stellar, Stress and the individual. Mechanisms leading to disease, Arch Intern Med 153(18) (1993) 2093-101.

[14] B.S. McEwen, Allostasis and allostatic load: implications for neuropsychopharmacology, Neuropsychopharmacology 22(2) (2000) 108-24.

[15] M.A. Arroll, Allostatic overload in myalgic encephalomyelitis/chronic fatigue syndrome (ME/CFS), Med Hypotheses 81(3) (2013) 506-8.

[16] A.S. Papadopoulos, A.J. Cleare, Hypothalamic-pituitary-adrenal axis dysfunction in chronic fatigue syndrome, Nat Rev Endocrinol 8(1) (2011) 22-32.

[17] S. Kempke, P. Luyten, S. De Coninck, B. Van Houdenhove, L.C. Mayes, S. Claes, Effects of early childhood trauma on hypothalamic-pituitary-adrenal (HPA) axis function in patients with Chronic Fatigue Syndrome, Psychoneuroendocrinology 52 (2015) 14-21.

[18] B. Van Houdenhove, F.P. Luyten, S. Kempke, Chronic fatigue syndrome/fibromyalgia: a "stressadaptation" model, Fatigue: Biomedicine, Health \& Behavior 1(3) (2013) 137-147. 
[19] S.M. Smith, W.W. Vale, The role of the hypothalamic-pituitary-adrenal axis in neuroendocrine responses to stress, Dialogues Clin Neurosci 8(4) (2006) 383-95.

[20] J. Gaab, D. Huster, R. Peisen, V. Engert, T. Schad, T.H. Schurmeyer, U. Ehlert, Low-dose dexamethasone suppression test in chronic fatigue syndrome and health, Psychosom Med 64(2) (2002) $311-8$

[21] F. Van Den Eede, G. Moorkens, W. Hulstijn, B. Van Houdenhove, P. Cosyns, B.G.C. Sabbe, S.J. Claes, Combined dexamethasone/corticotropin-releasing factor test in chronic fatigue syndrome, Psychological medicine 38(7) (2008) 963-73.

[22] A. Papadopoulos, M. Ebrecht, A.D. Roberts, L. Poon, N. Rohleder, A.J. Cleare, Glucocorticoid receptor mediated negative feedback in chronic fatigue syndrome using the low dose $(0.5 \mathrm{mg})$ dexamethasone suppression test, J Affect Disord 112(1-3) (2009) 289-94.

[23] R. Yehuda, J.D. Flory, L.M. Bierer, C. Henn-Haase, A. Lehrner, F. Desarnaud, I. Makotkine, N.P. Daskalakis, C.R. Marmar, M.J. Meaney, Lower methylation of glucocorticoid receptor gene promoter 1F in peripheral blood of veterans with posttraumatic stress disorder, Biol Psychiatry 77(4) (2015) 35664.

[24] E. Vangeel, F. Van Den Eede, T. Hompes, B. Izzi, J. Del Favero, G. Moorkens, D. Lambrechts, K. Freson, S. Claes, Chronic Fatigue Syndrome and DNA Hypomethylation of the Glucocorticoid Receptor Gene Promoter 1F Region: Associations With HPA Axis Hypofunction and Childhood Trauma, Psychosom Med 77(8) (2015) 853-62.

[25] H. Palma-Gudiel, A. Cordova-Palomera, J.C. Leza, L. Fananas, Glucocorticoid receptor gene (NR3C1) methylation processes as mediators of early adversity in stress-related disorders causality: A critical review, Neurosci Biobehav Rev 55 (2015) 520-35. 
[26] P.O. McGowan, A. Sasaki, A.C. D'Alessio, S. Dymov, B. Labonté, M. Szyf, G. Turecki, M.J. Meaney, Epigenetic regulation of the glucocorticoid receptor in human brain associates with childhood abuse, Nature neuroscience 12(3) (2009) 342-8.

[27] N. Perroud, A. Paoloni-Giacobino, P. Prada, E. Olié, A. Salzmann, R. Nicastro, S. Guillaume, D. Mouthon, C. Stouder, K. Dieben, P. Huguelet, P. Courtet, A. Malafosse, Increased methylation of glucocorticoid receptor gene (NR3C1) in adults with a history of childhood maltreatment: a link with the severity and type of trauma, Translational psychiatry 1 (2011) e59-e59.

[28] P.A. Melas, Y. Wei, C.C.Y. Wong, L.K. Sjöholm, E. Åberg, J. Mill, M. Schalling, Y. Forsell, C. Lavebratt, Genetic and epigenetic associations of MAOA and NR3C1 with depression and childhood adversities, The international journal of neuropsychopharmacology / official scientific journal of the Collegium Internationale Neuropsychopharmacologicum (CINP) 16(7) (2013) 1513-28.

[29] N. Saez-Francas, J. Alegre, N. Calvo, J. Antonio Ramos-Quiroga, E. Ruiz, J. Hernandez-Vara, M. Casas, Attention-deficit hyperactivity disorder in chronic fatigue syndrome patients, Psychiatry Res 200(2-3) (2012) 748-53

[30] C. Heim, D. Wagner, E. Maloney, D.A. Papanicolaou, L. Solomon, J.F. Jones, E.R. Unger, W.C. Reeves, Early adverse experience and risk for chronic fatigue syndrome: results from a populationbased study, Archives of general psychiatry 63(11) (2006) 1258-66.

[31] C. Heim, U.M. Nater, E. Maloney, R. Boneva, J.F. Jones, W.C. Reeves, Childhood trauma and risk for chronic fatigue syndrome: association with neuroendocrine dysfunction, Archives of general psychiatry 66(1) (2009) 72-80.

[32] F. Van Den Eede, T. Haccuria, M. De Venter, G. Moorkens, Childhood sexual abuse and chronic fatigue syndrome, The British journal of psychiatry : the journal of mental science 200(2) (2012) 1645. 
[33] J.R. Yancey, S.M. Thomas, Chronic fatigue syndrome: diagnosis and treatment, Am Fam Physician 86(8) (2012) 741-6.

[34] C. Clark, L. Goodwin, S.A. Stansfeld, M. Hotopf, P.D. White, Premorbid risk markers for chronic fatigue syndrome in the 1958 British birth cohort, Br J Psychiatry 199(4) (2011) 323-9.

[35] S.M. Collin, K. Tilling, C. Joinson, K.A. Rimes, R.M. Pearson, R.A. Hughes, J.A. Sterne, E. Crawley, Maternal and childhood psychological factors predict chronic disabling fatigue at age 13 years, J Adolesc Health 56(2) (2015) 181-7.

[36] E. Crawley, R. Hughes, K. Northstone, K. Tilling, A. Emond, J.A. Sterne, Chronic disabling fatigue at age 13 and association with family adversity, Pediatrics 130(1) (2012) e71-9.

[37] K.G. Raphael, C.S. Widom, Post-traumatic stress disorder moderates the relation between documented childhood victimization and pain 30 years later, Pain 152(1) (2011) 163-9.

[38] C.U. Rask, E. Ornbol, E.M. Olsen, P. Fink, A.M. Skovgaard, Infant behaviors are predictive of functional somatic symptoms at ages 5-7 years: results from the Copenhagen Child Cohort CCC2000, J Pediatr 162(2) (2013) 335-42.

[39] J.C. Ulirsch, L.E. Ballina, A.C. Soward, C. Rossi, W. Hauda, D. Holbrook, R. Wheeler, K.A. Foley, J. Batts, R. Collette, E. Goodman, S.A. McLean, Pain and somatic symptoms are sequelae of sexual assault: results of a prospective longitudinal study, Eur J Pain 18(4) (2014) 559-66.

[40] A. van Gils, K.A. Janssens, J.G. Rosmalen, Family disruption increases functional somatic symptoms in late adolescence: the TRAILS study, Health Psychol 33(11) (2014) 1354-61.

[41] C. Anacker, K.J. O'Donnell, M.J. Meaney, Early life adversity and the epigenetic programming of hypothalamic-pituitary-adrenal function, Dialogues Clin Neurosci 16(3) (2014) 321-33. 
[42] M.B. First, R.L. Spitzer, M.L. Gibbon, J.B.W. Williams, The structured clinical interview for DSM-IV Axis I Disorders (SCID-I), research version, Biometric Research, New York State Psychiatric Institute, New York, NY, 1996.

[43] J.H. Vercoulen, C.M. Swanink, J.F. Fennis, J.M. Galama, J.W. van der Meer, G. Bleijenberg, Dimensional assessment of chronic fatigue syndrome, J Psychosom Res 38(5) (1994) 383-92.

[44] D.P. Bernstein, J.A. Stein, M.D. Newcomb, E. Walker, D. Pogge, T. Ahluvalia, J. Stokes, L. Handelsman, M. Medrano, D. Desmond, W. Zule, Development and validation of a brief screening version of the Childhood Trauma Questionnaire, Child Abuse Negl 27(2) (2003) 169-90.

[45] Heim C, Nater UM, Maloney E, Boneva R, Jones JF, Reeves WC: Childhood trauma and risk for chronic fatigue syndrome: association with neuroendocrine dysfunction. Archives of general psychiatry 2009, 66:72-80.

[46] A.S. Zigmond, R.P. Snaith, The hospital anxiety and depression scale, Acta Psychiatr Scand 67(6) (1983) 361-70.

[47] B. Izzi, A.M. Binder, K.B. Michels, Pyrosequencing Evaluation of Widely Available Bisulfite Conversion Methods: Considerations for Application, Med Epigenet 2(1) (2014) 28-36.

[48] H.E. Suchiman, R.C. Slieker, D. Kremer, P.E. Slagboom, B.T. Heijmans, E.W. Tobi, Design, measurement and processing of region-specific DNA methylation assays: the mass spectrometrybased method EpiTYPER, Front Genet 6 (2015) 287.

[49] Schur RR, Boks MP, Rutten BPF, Daskalakis NP, de Nijs L, van Zuiden M, Kavelaars A, Heijnen CJ, Joels M, Kahn RS, et al: Longitudinal changes in glucocorticoid receptor exon $1 \mathrm{~F}$ methylation and psychopathology after military deployment. Transl Psychiatry 2017, 7:e1181.

[50] Fritz CO, Morris PE, Richler JJ: Effect size estimates: current use, calculations, and interpretation. J Exp Psychol Gen 2012, 141:2-18. 
[51] A. Martin-Blanco, M. Ferrer, J. Soler, J. Salazar, D. Vega, O. Andion, C. Sanchez-Mora, M.J. Arranz, M. Ribases, A. Feliu-Soler, V. Perez, J.C. Pascual, Association between methylation of the glucocorticoid receptor gene, childhood maltreatment, and clinical severity in borderline personality disorder, J Psychiatr Res 57 (2014) 34-40.

[52] S. Edelman, I. Shalev, F. Uzefovsky, S. Israel, A. Knafo, I. Kremer, D. Mankuta, M. Kaitz, R.P. Ebstein, Epigenetic and genetic factors predict women's salivary cortisol following a threat to the social self, PloS one 7(11) (2012) e48597-e48597.

[53] R. Yehuda, R.K. Yang, M.S. Buchsbaum, J.A. Golier, Alterations in cortisol negative feedback inhibition as examined using the ACTH response to cortisol administration in PTSD, Psychoneuroendocrinology 31(4) (2006) 447-51.

[54] R. Yehuda, N.P. Daskalakis, F. Desarnaud, I. Makotkine, A.L. Lehrner, E. Koch, J.D. Flory, J.D. Buxbaum, M.J. Meaney, L.M. Bierer, Epigenetic Biomarkers as Predictors and Correlates of Symptom Improvement Following Psychotherapy in Combat Veterans with PTSD, Frontiers in psychiatry 4 (2013) 118-118.

[55] W.C. de Vega, S.D. Vernon, P.O. McGowan, DNA methylation modifications associated with chronic fatigue syndrome, PLoS One 9(8) (2014) e104757.

[56] E.W. Brenu, D.R. Staines, S.M. Marshall-Gradisnik, Methylation Profile of CD4+ T Cells in Chronic Fatigue Syndrome/Myalgic Encephalomyelitis, J Clin Cell Immunol 5 (2014).

[57] B. Van Houdenhove, P. Luyten, U.T. Egle, The role of childhood trauma in chronic pain and fatigue, in: V.L. Banyard, V.J. Edwards, K.A. Kendall-Tackett (Eds.), Trauma and physical health. Understanding the effects of extreme stress and of psychological harm, Routledge, London, England, 2009, pp. 37-64. [58] Breton CV, Marsit CJ, Faustman E, Nadeau K, Goodrich JM, Dolinoy DC, Herbstman J, Holland N, LaSalle JM, Schmidt R, et al: Small-Magnitude Effect Sizes in Epigenetic End Points are Important in 
Children's Environmental Health Studies: The Children's Environmental Health and Disease Prevention Research Center's Epigenetics Working Group. Environ Health Perspect 2017, 125:511-526.

[59] B. Labonté, N. Azoulay, V. Yerko, G. Turecki, A. Brunet, Epigenetic modulation of glucocorticoid receptors in posttraumatic stress disorder, Translational psychiatry 4 (2014) e368-e368.

[60] A.R. Tyrka, L.H. Price, C. Marsit, O.C. Walters, L.L. Carpenter, Childhood adversity and epigenetic modulation of the leukocyte glucocorticoid receptor: preliminary findings in healthy adults, PloS one 7(1) (2012) e30148-e30148.

[61] N. Perroud, E. Rutembesa, A. Paoloni-Giacobino, J. Mutabaruka, L. Mutesa, L. Stenz, A. Malafosse, F. Karege, The Tutsi genocide and transgenerational transmission of maternal stress: epigenetics and biology of the HPA axis, The world journal of biological psychiatry : the official journal of the World Federation of Societies of Biological Psychiatry 15(4) (2014) 334-45.

[62] N. Weder, H. Zhang, K. Jensen, B.Z. Yang, A. Simen, A. Jackowski, D. Lipschitz, H. DouglasPalumberi, M. Ge, F. Perepletchikova, K. O'Loughlin, J.J. Hudziak, J. Gelernter, J. Kaufman, Child abuse, depression, and methylation in genes involved with stress, neural plasticity, and brain circuitry, Journal of the American Academy of Child and Adolescent Psychiatry 53(4) (2014) 417-424.e5.

[63] T.F. Oberlander, J. Weinberg, M. Papsdorf, R. Grunau, S. Misri, A.M. Devlin, Prenatal exposure to maternal depression, neonatal methylation of human glucocorticoid receptor gene (NR3C1) and infant cortisol stress responses, Epigenetics 3(2) (2008) 97-106. 
Table 1. Descriptives of the Chronic Fatigue Syndrome (CFS) patient sample $(n=80)$.

\begin{tabular}{llc}
\hline Age (years) & $M$ (range) & $41.36(18-61)$ \\
S.D. & 10.73 \\
Duration of illness (months) & $M$ (range) & $67.13(3-360)$ \\
S.D. & 73.82 \\
Level of education & Primary education & $2(2.5 \%)$ \\
& Lower secondary education & $7(8.8 \%)$ \\
& Higher secondary education & $28(35 \%)$ \\
Undergraduate degree & $37(46.2 \%)$ \\
Depressive symptoms (HADS) & University degree & $6(7.5 \%)$ \\
& $M$ (range) & $8.75(1-20)$ \\
& S.D. & 4.17
\end{tabular}

Mean, M; standard deviation, S.D.; Hospital Anxiety and Depression Scale, HADS 
Table 2. Frequency and valid percent of childhood trauma in the CFS patient group $(n=78)$

childhood trauma

emotional neglect

$30(38.5 \%)$

emotional abuse

$27(34.6 \%)$

sexual abuse

$21(26.9 \%)$

physical neglect

$13(16.7 \%)$

physical abuse

$7(9 \%)$

Total CTQ

$40(51.3 \%)$ 


\begin{tabular}{|c|c|c|c|c|c|c|c|}
\hline \multirow[b]{3}{*}{ Pyrosequencing } & \multicolumn{2}{|c|}{ Controls } & \multicolumn{2}{|c|}{ CFS } & \multirow[b]{2}{*}{$\begin{array}{c}\text { Percent } \\
\text { methylation } \\
\text { difference }\end{array}$} & \multirow[b]{2}{*}{$\begin{array}{l}\text { 2-tailed } \mathrm{P} \text { - } \\
\text { value }\end{array}$} & \multirow[b]{2}{*}{$\begin{array}{c}\text { Effect } \\
\text { size } \\
\text { estimate }\end{array}$} \\
\hline & Mean & Std Error & Mean & Std Error & & & \\
\hline & & & & & & & \\
\hline CpG_1 & 1.12 & 0.06 & 0.96 & 0.04 & -0.16 & $0.010^{*}$ & 0.206 \\
\hline CpG_2 & 1.51 & 0.02 & 1.55 & 0.04 & 0.04 & 0.884 & 0.012 \\
\hline CpG_3 & 1.08 & 0.04 & 1.09 & 0.05 & 0.01 & 0.580 & 0.044 \\
\hline CpG_4 & 1.50 & 0.04 & 1.43 & 0.04 & -0.07 & $0.017^{*}$ & 0.189 \\
\hline CpG_5 & 2.06 & 0.05 & 2.01 & 0.05 & -0.05 & 0.190 & 0.104 \\
\hline CpG_6 & 0.84 & 0.02 & 0.77 & 0.03 & -0.08 & $0.003 * *$ & 0.240 \\
\hline CpG_7 & 2.36 & 0.04 & 2.23 & 0.03 & -0.13 & $0.006 * *$ & 0.216 \\
\hline CpG_8 & 1.51 & 0.04 & 1.19 & 0.03 & -0.32 & $<0.0001 * *$ & 0.558 \\
\hline \multicolumn{8}{|l|}{ EpiTYPER } \\
\hline CpG_9 & 0.77 & 0.29 & 0.98 & 0.31 & 0.21 & 0.140 & 0.118 \\
\hline CpG_10.11 & 8.03 & 0.63 & 8.29 & 0.98 & 0.26 & 0.267 & 0.089 \\
\hline CpG_12.13 & 2.83 & 0.09 & 2.88 & 0.09 & 0.04 & 0.932 & 0.007 \\
\hline CpG_14 & 0.37 & 0.08 & 0.22 & 0.05 & -0.15 & 0.263 & 0.087 \\
\hline CpG_17.18 & 0.83 & 0.06 & 0.76 & 0.05 & -0.07 & 0.455 & 0.058 \\
\hline CpG_19 & 0.60 & 0.07 & 0.45 & 0.04 & -0.15 & 0.261 & 0.087 \\
\hline CpG_20.21 & 2.04 & 0.11 & 2.11 & 0.11 & 0.07 & 0.411 & 0.063 \\
\hline CpG_36 & 1.65 & 0.09 & 1.76 & 0.12 & 0.12 & 0.606 & 0.040 \\
\hline CpG_38.39 & 1.24 & 0.08 & 1.29 & 0.06 & 0.06 & 0.300 & 0.080 \\
\hline CpG_47 & 0.11 & 0.04 & 0.14 & 0.06 & 0.03 & 0.531 & 0.049 \\
\hline Average NR3C1 & 1.67 & 0.04 & 1.69 & 0.08 & 0.02 & 0.398 & 0.065 \\
\hline
\end{tabular}




\section{Functional}

methylation

1.46

0.03

1.37

0.03

$-0.09$

$0.003^{* *}$

0.231

NR3C1-1F DNA methylation was assessed using pyrosequencing for the region spanning CpG_1 - CpG_8 and EpiTYPER for the region spanning CpG_9 -

CpG_47. Mann-Whitney U tests, *: significant $p$-value $<0.05 ;{ }^{* *}$ : significant $p$-value $<0.01$ 